\title{
Day-to-Day Home Blood Pressure Variability is Associated with Cerebral Small Vessel Disease Burden in a Memory Clinic Population
}

\author{
Rianne A.A. de Heus ${ }^{\mathrm{a}}$, Stacha F.I. Reumers ${ }^{\mathrm{a}}$, Alba van der Have ${ }^{\mathrm{a}}$, Maxime Tumelaire ${ }^{\mathrm{a}}$, \\ Phillip J. Tully ${ }^{\mathrm{b}}$ and Jurgen A.H.R. Claassen ${ }^{\mathrm{a}, *}$ \\ ${ }^{a}$ Radboud University Medical Center, Donders Institute for Brain Cognition and Behaviour, \\ Department of Geriatric Medicine, Radboudumc Alzheimer Center, Nijmegen, The Netherlands \\ ${ }^{\mathrm{b}}$ School of Medicine, The University of Adelaide, Australia
}

Handling Associate Editor: Ronan O’Caoimh

Accepted 7 January 2020

\begin{abstract}
.
Background: High visit-to-visit blood pressure variability (BPV) has been associated with cognitive decline and cerebral small vessel disease (cSVD), in particular cerebrovascular lesions. Whether day-to-day BPV also relates to cSVD has not been investigated.

Objective: To investigate the cross-sectional association between day-to-day BPV and total cSVD MRI burden in older memory clinic patients.

Methods: We included outpatients referred to our memory clinic, who underwent cerebral MRI as part of their diagnostic assessment. We determined the validated total cSVD score (ranging from 0-4) by combining four markers of cSVD that were visually rated. Home blood pressure (BP) measurements were performed for one week, twice a day, according to international guidelines. BPV was defined as the within-subject coefficient of variation (CV; standard deviation/mean BP*100). We used multivariable ordinal logistic regression analyses adjusted for age, sex, smoking, diabetes, antihypertensive medication, history of cardiovascular disease, and mean BP.

Results: For 82 patients (aged 71.2 \pm 7.9 years), mean home BP was 140/79 $\pm 15 / 9 \mathrm{mmHg}$. Dementia and mild cognitive impairment were diagnosed in $46 \%$ and $34 \%$, respectively. $78 \%$ had one or more markers of cSVD. Systolic CV was associated with cSVD burden (adjusted odds ratio per point increase in CV $=1.29,95 \%$ confidence interval $=1.04-1.60, p=0.022$ ). There were no differences in diastolic CV and mean BP between the cSVD groups. When we differentiated between morning and evening BP, only evening BPV remained significantly associated with total cSVD burden.

Conclusion: Day-to-day systolic BPV is associated with cSVD burden in memory clinic patients. Future research should indicate whether lowering BPV should be included in BP management in older people with memory complaints.
\end{abstract}

Keywords: Blood pressure, blood pressure variability, cerebrovascular damage, cognition, dementia, home monitoring, small vessel disease

\footnotetext{
*Correspondence to: Jurgen Claassen, MD, PhD, Department of Geriatric Medicine, Radboud University Medical Center, P.O.
} 


\section{INTRODUCTION}

Cerebral small vessel disease (cSVD) is the umbrella term for a range of abnormalities of the small vessels of the brain, including arterioles, capillaries, and venules [1]. Individual neuroimaging markers of cSVD include lacunes, white matter hyperintensities (WMH), cerebral microbleeds (CMB), and enlarged perivascular spaces (ePVS) in the basal ganglia. These markers likely share similar pathology and are common, often asymptomatic, findings on magnetic resonance imaging (MRI) in older people [2, 3]. Clinically, they are associated with an increased risk of stroke, gait disturbances, and cognitive decline [4]. It is postulated that CSVD pathology contributes to up to $45 \%$ of all dementia cases $[5,6]$.

While the exact pathogenesis of cSVD is still unclear, it is evident that cardiovascular health plays an important role. Hypertension is associated with each individual marker of cSVD [7-10]. High blood pressure (BP) is thought to affect brain health by causing structural changes to the cerebral microvasculature that lead to endothelial dysfunction, increased permeability of the blood-brain barrier and dysregulation of cerebral blood flow [11, 12]. Clinically, it is evident that hypertension during midlife is a risk factor for developing dementia [13]. However, several ambiguities remain. For example, contradictions exist on the role of BP in older people who already present with some degree of cognitive impairment [14], and on the effect of BP lowering in order to reduce dementia risk [15]. The conflicting findings regarding mean BP level make a case for looking beyond the use of BP as a static measurement only and for taking into account the dynamic aspects of BP.

Within the cardiovascular field there is increasing attention to study the prognostic significance of BP variability (BPV) in relation to adverse health outcomes and organ damage, including the brain [16]. High BPV is associated with stroke and transient ischemic attack, mortality, and cardiovascular outcomes [17-19]. There is limited information on BPV and cSVD markers, other than WMH [20, 21], but it is hypothesized that high fluctuations in BP put more stress on vessel walls than high BP only, accelerating the hypertension-cSVD pathology cascade.

It is likely that the causes and consequences of high BPV depend on which timeframe is investigated [22]. Previous studies reporting an association between BPV and markers of cSVD focused on either long-term BPV, based on consecutive BP measurements performed over multiple office visits in a period of months or years, or on short-term BPV, based on 24-h ambulatory BP measurements (ABPM) with a $15-30$ min interval. Home BP measurements (HBPM), taken in the morning and evening over a week, have the advantage of providing information on the consistency of BP control on a day-to-day basis [23], but have not been widely studied in association with cSVD burden [24].

The majority of the previous studies on BPV and cSVD focused on only one marker of cSVD. Cooccurrence of different markers of cSVD is associated with decreased cognitive function, indicating that the markers have additive effects when present together [27]. The 'total cSVD score' combines the individual markers into one score to reflect overall burden [28]. This score has been associated with reduced cognitive function in a memory clinic population [29], a population in which substantial burden of CSVD is expected.

Taken together, this study adds to previous work by assessing the association between BPV determined on a day-to-day basis, using HBPM, and the total burden of cSVD. Specifically, the aim of this study was to investigate the cross-sectional association between day-to-day BPV and total cSVD score in older people attending a memory clinic.

\section{METHODS}

\section{Study design and participants}

In this cross-sectional study, we included a convenience sample made up of people aged $>50$ years who were referred to the memory clinic of the Radboud university medical center (Nijmegen, The Netherlands). Inclusion lasted from January 2014 until December 2018. In order to be eligible, participants should have performed HBPM and had a cerebral MRI scan as part of their diagnostic assessment. At the end of their first visit to the memory clinic, participants were asked to perform HBPM by their clinician or nurse. Whether an MRI scan was deemed necessary for diagnosis was decided in a multidisciplinary meeting. For example, when the diagnosis was already clear from the clinical information available, an MRI was not performed. This was based on previous research in our memory clinic on the added diagnostic value of MRI [30]. In the inclusion period, a total of 1,310 patients were seen at the memory clinic, 327 of whom had 
an MRI performed. Thirty-five percent of this group also performed HBPM. Participants with a clinical history of stroke or who were diagnosed with a neurological condition other than a neurodegenerative disease were excluded from the current analyses. The study was submitted to the Medical Ethics Committee (CMO Arnhem-Nijmegen) and was exempt from formal approval because the study did not fall within the remit of the "Medical Research Involving Human Subjects Act". The study was also exempt from the need to obtain explicit written informed consent, because of the low additional burden of HBPM. Despite this, oral informed consent was asked from each participant and, if applicable, their caregiver.

\section{Day-to-day blood pressure variability}

Participants performed HBPM using a validated, memory-equipped, automatic oscillometric device (Microlife WatchBP Home, Microlife, Heerbrugg, Switzerland) [31], following an international recommended protocol [32]. They received oral and written instructions and on how to perform HBPM and could be aided by their caregiver (if available). The protocol comprised duplicate measurements in the morning (5.00-11.00 am) and evening (5.00-11.00 pm) for 7 consecutive days, resulting in a maximum of 28 measurements. Measurements of day 1 were discarded and duplicate measurements were averaged, to reduce variation resulting from random or measurement error. A minimum of 8 (out of 12) measurements was set as a requirement to be included in the analysis. For separate analysis of morning and evening measure- ments, 4 (out of 6) was the minimum required number of measurements. Day-to-day BPV was defined as the within-subject coefficient of variation $(\mathrm{CV})$ of both systolic (SBP) and diastolic blood pressure (DBP), calculated by dividing the standard deviation (SD) with the mean $(\mathrm{CV}=\mathrm{SD} /$ mean*100\%).

\section{Total cerebral small vessel disease score}

Participants were scanned with either of two scanners, Magnetom Avanto, Siemens Healthcare (1.5T) or Magnetom Prisma, Siemens Healtcare (3.0T). The scan protocol included T1-weighted, T2-weighted, fluid-attenuated inversion recovery (FLAIR) and susceptibility-weighted imaging (SWI) or $\mathrm{T} 2 *$-weighted gradient-echo (GRE) sequences. Details of the scan parameters are provided in Supplementary Table 1 . The total cSVD score consisted of a previously described four-point scale [28], including presence of $\mathrm{WMH}, \mathrm{CMB}$, lacunes, and ePVS in the basal ganglia (Fig. 1). Two trained clinicians rated WHM severity and presence of CMBs (AH, MT). WMH severity was rated on FLAIR images using the Fazekas scale [33]. One point was added to the cSVD score in case of severe $\mathrm{WMH}$, defined as periventricular WMH Fazekas grade 3 or deep WMH Fazekas grade 2 or 3. CMBs were rated on the SWI or T2* GRE images using the Microbleed Anatomical Rating Scale [34]. One point was added to the cSVD score if one or more microbleeds were present. Interrater kappa was $\kappa=0.90$ for presence of severe $\mathrm{WMH}$ and $\kappa=0.66$ for presence of CMBs. Lacunes of presumed vascular origin were identified

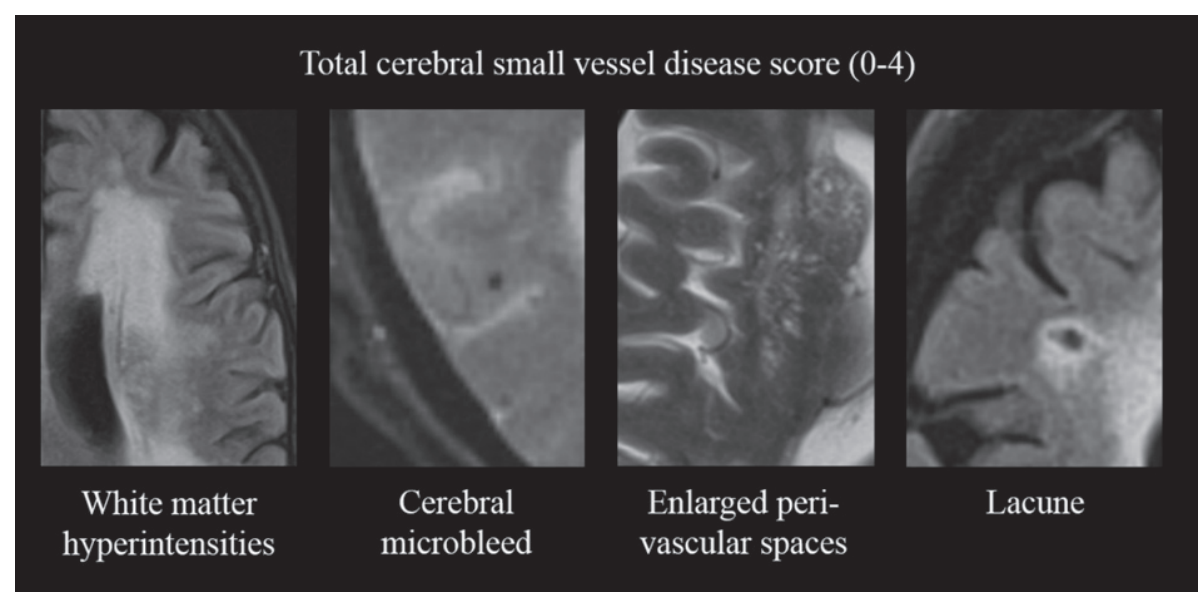

Fig. 1. Construction of the total cerebral small vessel disease score. One point is added to the score in case of presence of: severe WMH (periventricular Fazekas grade 3 or deep Fazekas grade $\geq 2$ ); $\geq 1$ cerebral microbleed; $>10$ perivascular spaces visible in the basal ganglia on at least one side of the brain; $\geq 1$ lacune. WMH, white matter hyperintensities. 
using FLAIR, T1-, and T2-weighted images by two raters (RH, SR). They were defined according to STandards for ReportIng Vascular changes on $\mathrm{nEu}-$ roimaging (STRIVE) criteria, as subcortical, round or ovoid shaped, fluid-filled cavities, 3-15 mm in diameter [1]. The presence of one or more lacunes resulted in one extra point on the cSVD score. ePVS in the basal ganglia were assessed using FLAIR and T2weighted images using the protocol of Doubal et al. (by RH, SR) [35]. One point was added to the cSVD score if $>10$ ePVS were visible in the basal ganglia on one side of the brain. Interrater kappa for presence of lacunes and for $>10 \mathrm{ePVS}$ was $\kappa=0.63$ and $\kappa=0.75$, respectively. In case of disagreement on any of the markers, a third rater (JC) assessed the images in order to achieve consensus. All rating was performed blinded to clinical details.

\section{Clinical assessment}

Additional information on descriptives and covariates was extracted from the patient's medical record and included age, gender, education, medical history, current medication use, body mass index (BMI), smoking status, office BP, and cognitive diagnosis. Office BP was measured in supine position using a manual sphygmomanometer. Cardiovascular history was marked as present if one of the following conditions were identified in the medical history of the patients' health record: chronic heart failure, coronary heart disease, arrhythmia, or peripheral artery disease. The cognitive diagnosis was based on all information from the geriatric assessment, including the cerebral MRI, and was established in a multidisciplinary meeting with geriatricians and neuropsychologists using international criteria [36, 37]. For descriptive purposes, we categorized participants into four groups: dementia (mild stage, any type), mild cognitive impairment, subjective cognitive impairment, and other diagnoses (i.e., psychiatric disorder).

\section{Statistical analyses}

Characteristics are presented as mean and standard deviation for continuous variables and percentage and frequency for categorical variables. The total cSVD score was used as an ordinal variable. Similar to previous work [38], cSVD scores of 3 and 4 were combined because each score had only few cases ( $n=16$ and $n=7$, respectively), resulting in a maximum score of 3 . Analysis of variance and chi-squared tests were used to compare characteristics by cSVD score. Ordinal logistic regression was used to investigate if indices of home BP were associated with cSVD score. The proportional odds assumption was checked through the test of parallel lines and no violations were identified. BP indices that were assessed were $\mathrm{CV}$ and mean of SBP and DBP. In the first model we adjusted for age and sex. In the second model, we additionally adjusted for current smoking, diabetes, use of antihypertensive medication and history of cardiovascular diseases (CVD). Since we found no evidence for multicollinearity between mean and $\mathrm{CV}$ of home BP (variance inflation factors all $<1.05$ ), we fitted a third model in which $\mathrm{CV}$ and mean $\mathrm{BP}$ were simultaneously added as predictors. All models were run separately for morning and evening BP indices. Additionally, we explored associations of BP indices with a dichotomized cSVD score $(0$ versus 1-4) and with individual markers of cSVD, using logistic regression analyses. Odds ratio's (OR) with 95\% confidence intervals (CI) are reported. For CV, OR are reported per unit increase. For mean BP, OR are reported per 10 (systolic) or 5 (diastolic) $\mathrm{mmHg}$ increase. All analyses were performed using IBM SPSS Statistics 25.0 (SPSS, Inc., Chicago, IL, USA).

\section{RESULTS}

\section{Characteristics}

In total, 104 participants met the inclusion criteria. Fifteen participants were excluded because of invalid HBPM data (too few measurements) and seven because of insufficient MRI quality, leaving 82 participants enrolled in the analysis. Mean age of the study population was $71.2 \pm 7.9$ years and $34 \%(n=28)$ were female. Dementia (mild stage) was diagnosed in $46 \%(n=38)$, mild cognitive impairment in $34 \%$ $(n=28)$, and $17 \%(n=14)$ were classified as having subjective memory complaints. In addition, two participants were diagnosed with a depression.

Twenty-two percent of the sample had no markers of cSVD, $24 \%$ had one marker, $26 \%$ had two markers, and $28 \%$ had three or four markers. Supplementary Figure 1 shows the occurrence of combinations of cSVD markers. The cSVD score did not differ between the different MR scanners (Supplementary Table 2). Characteristics of the total sample and according to cSVD score are presented in Table 1. Age increased with increasing cSVD score $(p<0.001)$. The prevalence of dementia increased with increasing CSVD scores, with a prevalence of 
Table 1

Characteristics of the total study sample and according to cSVD score

\begin{tabular}{|c|c|c|c|c|c|c|}
\hline Variable & Total & cSVD 0 & $\mathrm{cSVD} 1$ & cSVD 2 & cSVD $3 / 4$ & ${ }^{*} p$-value \\
\hline $\mathrm{n}$ & 82 & 18 & 20 & 21 & 23 & \\
\hline Age (y) & $71.2 \pm 7.9$ & $64.7 \pm 7.8$ & $68.9 \pm 5.5$ & $73.6 \pm 5.6$ & $76.2 \pm 7.6$ & $<0.001$ \\
\hline Sex (\% female) & $28(34.1)$ & $5(27.8)$ & $7(35.0)$ & $9(42.9)$ & $7(30.4)$ & 0.758 \\
\hline Living with spouse $(\%)$ & $60(73.2)$ & $14(77.8)$ & $18(90.0)$ & $12(57.1)$ & $16(69.6)$ & 0.113 \\
\hline Education (y) & $11.0 \pm 2.8$ & $10.9 \pm 2.8$ & $11.7 \pm 2.8$ & $11.6 \pm 3.4$ & $11.3 \pm 2.9$ & 0.771 \\
\hline BMI $\left(\mathrm{kg} / \mathrm{m}^{2}\right)^{* *}$ & $25.7 \pm 4.1$ & $27.3 \pm 5.4$ & $27.1 \pm 4.2$ & $23.8 \pm 3.2$ & $24.9 \pm 3.0$ & 0.031 \\
\hline History of CVD (\%) & $24(29.3)$ & $8(44.4)$ & $7(35.0)$ & $5(23.8)$ & $4(17.4)$ & 0.242 \\
\hline Diabetes $(\%)$ & $10(12.2)$ & $2(11.1)$ & $1(5.0)$ & $1(4.8)$ & $6(26.1)$ & 0.102 \\
\hline Current smoking (\%) & $12(14.6)$ & $4(22.2)$ & 0 & $5(23.8)$ & $3(13.0)$ & 0.126 \\
\hline Antihypertensives (\%) & $40(48.8)$ & $10(55.6)$ & $8(40.0)$ & $8(38.1)$ & $14(60.9)$ & 0.354 \\
\hline Diagnosis $(\%)$ & & & & & & 0.039 \\
\hline Dementia & $38(46.3)$ & $5(27.8)$ & $6(30.0)$ & $10(47.6)$ & $17(73.9)$ & \\
\hline MCI & $28(34.1)$ & $7(38.9)$ & $7(35.0)$ & $10(47.6)$ & $4(17.4)$ & \\
\hline SCI & $14(17.1)$ & $5(27.8)$ & $6(30.0)$ & $1(4.8)$ & $2(8.7)$ & \\
\hline Other & $2(2.4)$ & $1(5.6)$ & $1(5.0)$ & 0 & 0 & \\
\hline MR scanner $(\% 3 \mathrm{~T})^{* * *}$ & $32(39.0)$ & $6(33.3)$ & $8(40.0)$ & $9(42.9)$ & $9(39.1)$ & 0.944 \\
\hline CSVD markers (\%) & & & & & & - \\
\hline Lacune(s) & $27(32.9)$ & - & $2(10.0)$ & $6(28.6)$ & $19(82.6)$ & \\
\hline WMH & $40(48.8)$ & - & $5(25.0)$ & $14(66.7)$ & $21(91.3)$ & \\
\hline $\mathrm{CMB}$ & $26(31.7)$ & - & $5(25.0)$ & $4(19.0)$ & $17(73.9)$ & \\
\hline ePVS & $45(54.9)$ & - & $8(40.0)$ & $18(85.7)$ & $19(82.6)$ & \\
\hline
\end{tabular}

Results are presented as mean \pm standard deviation or number (frequency). cSVD, cerebral small vessel disease; BMI, body mass index; CVD, cardiovascular disease; MCI, mild cognitive impairment; SCI, subjective cognitive impairment; WMH, white matter hyperintensities; CMB, cerebral microbleeds; ePVS, enlarged perivascular space. * Resulting from univariate analysis of variance (continuous variables) or chi-square test (categorical variables).

** Available for $n=69$. ${ }^{* * *}$ Those not scanned on the 3T scanner were scanned on the $1.5 \mathrm{~T}$ scanner.

$28 \%$ in those with cSVD score of 0 , compared to $74 \%$ in those with cSVD score of $3 / 4(p=0.039)$.

\section{Blood pressure (variability) and cSVD score}

Mean home BP was $140 / 79 \pm 15 / 9 \mathrm{mmHg}$, while office BP was 160/85 $\pm 19 / 10 \mathrm{mmHg}$. Univariate analyses, presented in Table 2, showed that SBP was lowest in the group without CSVD burden and highest in the group with highest cSVD burden $(p<0.001$ for home SBP, $p=0.015$ for office SBP). The same trend was observed for DBP, but this was not statistically significant ( $p=0.294$ for home DBP, $p=0.074$ for office DBP).

Figure 2 shows the individual data points for systolic and diastolic CV by cSVD burden. Univariate analyses showed that there was a difference between the systolic CVs of the cSVD groups $(p=0.017)$. The diastolic $\mathrm{CV}$ was marginally different between groups $(p=0.125)$. Table 3 present the results from the multivariable ordinal regression models. Systolic $\mathrm{CV}$ was associated with $\mathrm{cSVD}$ burden $(\mathrm{OR}=1.32$, 95\% CI $=1.07-1.63, p=0.010$ ), after adjustment for age, sex, current smoking, diabetes, use of antihypertensive medication and history of CVD. This association remained significant when we additionally adjusted for mean systolic BP $(\mathrm{OR}=1.29,95 \%$
$\mathrm{CI}=1.04-1.60, p=0.022)$. Diastolic CV and mean BP were not associated with cSVD burden (Table 3).

As already suggested by Fig. 2, pairwise comparisons in the univariate analyses showed that those with no cSVD burden had a lower systolic CV compared to those with a cSVD score of 1,2 , or 3 , without differences between the latter three groups (Supplementary Table 3). Therefore, logistic regression analyses were performed comparing presence versus absence of cSVD burden. After adjustment for age, sex, current smoking, diabetes, antihypertensive medication, and history of CVD both systolic and diastolic $\mathrm{CV}$ were associated with presence of cSVD (systolic: $\mathrm{OR}=2.51,95 \% \mathrm{CI}=1.21-5.18, p=0.013$; diastolic: $\mathrm{OR}=1.48,95 \% \mathrm{CI}=1.03-2.13, p=0.036$ ). These associations remained significant when we additionally adjusted for mean BP. See Supplementary Table 4 for the results from all logistic regression models. Supplementary Table 5 presents the associations between BP parameters and the individual cSVD markers. All markers were individually associated with higher BPV.

\section{Morning and evening blood pressure (variability)}

Paired samples-t-test showed that BPV was higher in the evening compared to morning, for both systolic 
Table 2

Blood pressure values of the total study sample and according to cSVD score

\begin{tabular}{lcccccc}
\hline Variable & Total & cSVD 0 & cSVD 1 & cSVD 2 & cSVD 3/4 & $*$ p \\
\hline Office BP & & & & & & \\
SBP (mmHg) & $159.6 \pm 19.2$ & $148.9 \pm 19.0$ & $155.6 \pm 22.8$ & $164.4 \pm 11.9$ & $166.9 \pm 17.5$ & 0.015 \\
DBP (mmHg) & $84.6 \pm 10.1$ & $80.8 \pm 9.7$ & $82.1 \pm 10.4$ & $85.8 \pm 9.3$ & $88.4 \pm 9.8$ & 0.074 \\
Home BP & & & & & \\
Readings (nr)* & $21.7 \pm 2.9$ & $22.0 \pm 2.5$ & $20.8 \pm 2.8$ & $22.4 \pm 2.2$ & $21.5 \pm 3.8$ & 0.347 \\
Hypertension (\%) & $49(59.8)$ & $6(33.3)$ & $17(85.0)$ & $9(42.9)$ & $17(73.9)$ & 0.002 \\
SBP (mmHg) & $139.7 \pm 14.6$ & $131.3 \pm 14.3$ & $145.1 \pm 12.0$ & $134.6 \pm 11.0$ & $146.4 \pm 15.2$ & 0.001 \\
DBP (mmHg) & $79.1 \pm 8.8$ & $76.9 \pm 8.0$ & $81.5 \pm 7.4$ & $77.4 \pm 8.1$ & $80.3 \pm 10.8$ & 0.294 \\
CV SBP (\%) & $7.1 \pm 2.2$ & $5.7 \pm 1.4$ & $7.3 \pm 2.5$ & $7.3 \pm 2.1$ & $7.7 \pm 2.2$ & 0.017 \\
CV DBP (\%) & $7.3 \pm 2.7$ & $6.1 \pm 1.6$ & $8.0 \pm 3.2$ & $7.3 \pm 2.6$ & $7.8 \pm 2.9$ & 0.125 \\
Morning BP & & & & & \\
SBP (mmHg) & $139.7 \pm 15.2$ & $131.0 \pm 14.5$ & $146.9 \pm 13.5$ & $133.8 \pm 10.6$ & $145.6 \pm 15.8$ & 0.001 \\
DBP (mmHg) & $80.0 \pm 9.3$ & $77.8 \pm 8.0$ & $83.2 \pm 8.4$ & $78.3 \pm 8.3$ & $80.4 \pm 11.2$ & 0.249 \\
CV SBP (\%) & $5.9 \pm 2.3$ & $4.6 \pm 1.9$ & $6.2 \pm 2.7$ & $6.1 \pm 2.4$ & $6.4 \pm 2.0$ & 0.057 \\
CV DBP (\%) & $5.9 \pm 3.0$ & $5.2 \pm 1.8$ & $7.1 \pm 4.4$ & $5.4 \pm 2.6$ & $5.8 \pm 2.3$ & 0.185 \\
Evening BP & & & & & & \\
SBP (mmHg) & $139.9 \pm 15.1$ & $131.5 \pm 14.8$ & $141.5 \pm 12.2$ & $136.4 \pm 12.8$ & $149.0 \pm 15.2$ & 0.001 \\
DBP (mmHg) & $78.3 \pm 8.9$ & $76.0 \pm 8.5$ & $78.5 \pm 7.0$ & $77.3 \pm 8.7$ & $81.2 \pm 10.6$ & 0.292 \\
CV SBP (\%) & $7.2 \pm 2.9$ & $6.0 \pm 2.0$ & $6.5 \pm 2.7$ & $7.2 \pm 3.0$ & $8.6 \pm 3.3$ & 0.033 \\
CV DBP (\%) & $7.0 \pm 3.7$ & $5.7 \pm 2.7$ & $6.9 \pm 2.2$ & $7.0 \pm 3.5$ & $8.2 \pm 5.1$ & 0.211 \\
\hline
\end{tabular}

Results are presented as mean \pm standard deviation. cSVD, cerebral small vessel disease; BP, blood pressure; SBP, systolic blood pressure; DBP, diastolic blood pressure; $\mathrm{CV}$, coefficient of variation. ${ }^{*}$ Resulting from univariate analysis of variance. ${ }^{* *}$ After removal of day 1 . Maximum value 24 .
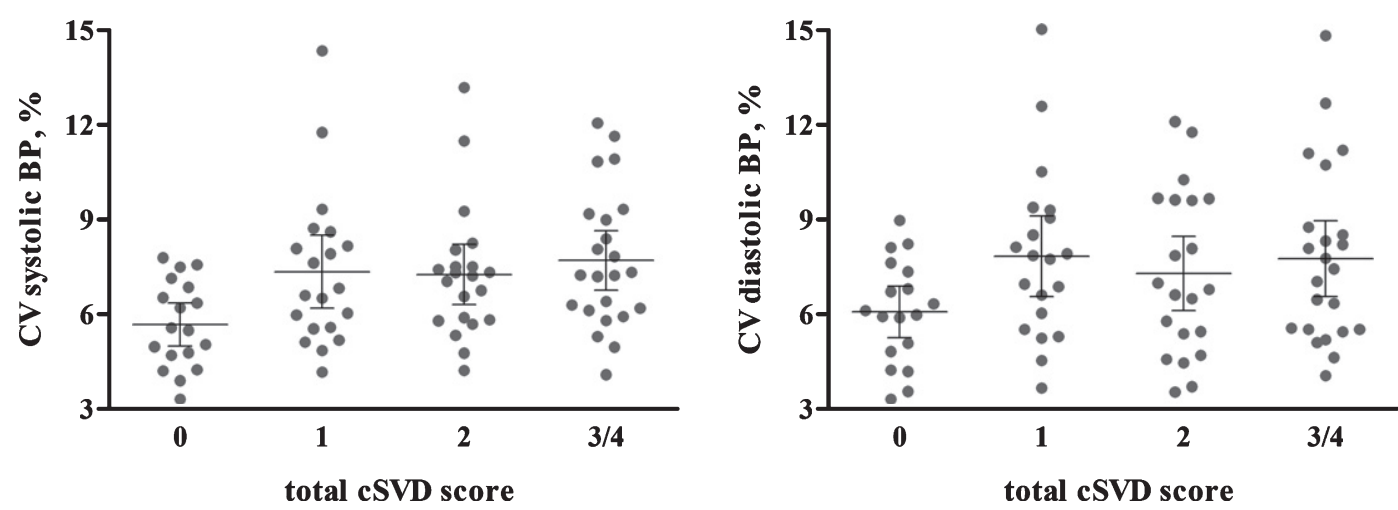

Fig. 2. Individual data points for systolic and diastolic CV by total cSVD score. Error bars present $95 \%$ confidence interval. $p$-value from univariate analysis of variance is 0.017 for systolic $\mathrm{CV}$ and 0.125 for diastolic $\mathrm{CV}$. BP, blood pressure; CV, coefficient of variation; cSVD, cerebral small vessel disease.

Table 3

Associations of total small vessel disease score with mean and coefficient of variation of home blood pressure

\begin{tabular}{lcccccc}
\hline Predictor & Model 1 & \multicolumn{3}{c}{ Model 2 } & Model 3 & \\
& OR $(95 \%$ CI $)$ & $p$ & OR $(95 \%$ CI $)$ & $p$ & OR (95\% CI) & $p$ \\
\hline Mean SBP & $1.32(0.98-1.77)$ & 0.066 & $1.28(0.94-1.73)$ & 0.114 & - & - \\
CV SBP & $1.24(1.02-1.52)$ & 0.034 & $1.32(1.07-1.63)$ & 0.010 & $1.29(1.04-1.60)$ & 0.022 \\
Mean DBP & $1.19(0.93-1.53)$ & 0.169 & $1.16(0.89-1.49)$ & 0.273 & - & - \\
CV DBP & $1.11(0.95-1.30)$ & 0.176 & $1.10(0.94-1.29)$ & 0.234 & $1.09(0.93-1.28)$ & 0.286 \\
\hline
\end{tabular}

Results from ordinal logistic regression. Model 1: adjusted for age and sex. Model 2: model 1+ current smoking, diabetes, use of antihypertensive medication and cardiovascular history. Model 3: model 2+ mean BP (systolic or diastolic). For mean BP, the OR is presented per 10 (systolic) or 5 (diastolic) $\mathrm{mmHg}$ increase. SBP, systolic blood pressure; DBP, diastolic blood pressure; OR, odds ratio; CI, confidence interval; CV, coefficient of variation. 
Table 4

Association of total small vessel disease score with mean and coefficient of variation of morning and evening home blood pressure

\begin{tabular}{|c|c|c|c|c|c|c|c|}
\hline \multirow[t]{2}{*}{ Predictor } & & \multicolumn{2}{|l|}{ Model 1} & \multicolumn{2}{|l|}{ Model 2} & \multicolumn{2}{|l|}{ Model 3} \\
\hline & & OR $(95 \% \mathrm{CI})$ & $p$ & OR $(95 \% \mathrm{CI})$ & $p$ & OR $(95 \% \mathrm{CI})$ & $p$ \\
\hline \multirow[t]{4}{*}{ Morning } & Mean SBP & $1.26(0.95-1.65)$ & 0.107 & $1.23(0.92-1.67)$ & 0.156 & - & - \\
\hline & CV SBP & $1.14(0.95-1.36)$ & 0.171 & $1.18(0.98-1.42)$ & 0.081 & $1.16(0.96-1.40)$ & 0.126 \\
\hline & Mean DBP & $1.14(0.90-1.44)$ & 0.256 & $1.11(0.87-1.43)$ & 0.367 & - & - \\
\hline & CV DBP & $1.01(0.88-1.16)$ & 0.875 & $1.01(0.88-1.16)$ & 0.918 & $1.00(0.87-1.15)$ & 0.995 \\
\hline \multirow[t]{4}{*}{ Evening* } & Mean SBP & $1.49(1.08-2.03)$ & 0.013 & $1.42(1.03-1.95)$ & 0.034 & - & - \\
\hline & CV SBP & $1.23(1.05-1.44)$ & 0.011 & $1.31(1.10-1.57)$ & 0.003 & $1.31(1.09-1.58)$ & 0.004 \\
\hline & Mean DBP & $1.32(1.01-1.73)$ & 0.048 & $1.25(0.95-1.66)$ & 0.113 & - & - \\
\hline & CV DBP & $1.16(1.01-1.33)$ & 0.033 & $1.15(0.99-1.33)$ & 0.051 & $1.15(0.99-1.32)$ & 0.062 \\
\hline
\end{tabular}

Results from ordinal logistic regression. Model 1: adjusted for age and sex. Model 2: model 1+current smoking, diabetes, use of antihypertensive medication and cardiovascular history. Model 3: model $2+$ mean BP (systolic or diastolic). For mean BP, the OR is presented per 10 (systolic) or 5 (diastolic) $\mathrm{mmHg}$ increase. $\mathrm{OR}$, odds ratio; $\mathrm{CI}$, confidence interval; $\mathrm{CV}$, coefficient of variation. ${ }^{*} n=79$, because of 3 participants with $<4$ duplo measurement in the evening.

CV (mean difference $=1.3,95 \% \quad \mathrm{CI}=0.6-2.1$, $p<0.001$ ) and diastolic CV (mean difference $=1.2$, 95\% CI $=0.2-2.2, p=0.025$ ). Separate regression analyses for morning and evening BP are presented in Table 4. Evening systolic CV $(\mathrm{OR}=1.31,95 \%$ $\mathrm{CI}=1.09-1.58, p=0.004)$ was associated with total cSVD score. Morning CV was not associated with total cSVD score. Mean evening SBP was associated with cSVD burden (OR per $10 \mathrm{mmHg}$ increase in $\mathrm{SBP}=1.42,95 \% \mathrm{CI}=1.03-1.95, p=0.034$ ).

\section{DISCUSSION}

In this cross-sectional study, we investigated the association between day-to-day BPV and total CSVD burden on MRI, in older adults who attended our memory clinic for cognitive evaluation. We found that higher systolic BPV was a risk factor for CSVD burden, after controlling for several confounders and mean SBP. Diastolic BPV or mean BP were not associated with cSVD burden. There does not seem to be a dose-response relationship between BPV and cSVD burden in these cross-sectional analyses, as no differences in BPV between scores of 1,2, and 3/4 were observed. When we separately calculated BPV for morning and evening measurements, only BPV in the evening was associated with the total cSVD score. This is, to the best of our knowledge, the first study investigating the association between BPV within a 1-week timeframe and total burden of cSVD.

Previous studies have mainly focused on the association between individual markers of CSVD and mainly measured BPV within other timeframes, i.e., from visit-to-visit or within 24-h. For example, several studies reported an association between WMH severity or progression and visit-to-visit BPV in healthy older adults [21, 39]. Only Yang et al. (2018) have reported the total cSVD score in relation to BPV [26]. These authors found that higher 24-h BPV was associated with cSVD burden in a healthy sample with a mean age of 68 years. Regarding day-to-day BPV, this has previously been linked to cognitive decline and progression of WMH in healthy people aged 80 years or older, with a mean MMSE of 25 and a low level of morbidities [24]. In two Japanese studies, the Hisayama study and Ohasama study, dayto-day BPV was associated with risk of dementia and cognitive decline, respectively [40,41]. We recently showed both visit-to-visit and day-to-day BPV were related to clinical progression of Alzheimer's disease [42], although for visit-to-visit BPV this was not replicated in another dementia cohort [43]. In the Maastricht study, a composite score comprising 24$\mathrm{h}$ and day-to-day BPV was associated with cognitive performance [44]. The current study adds to the previous work on day-to-day BPV, by providing evidence for the potential underlying mechanism linking BPV and cognition. Although not completely understood, the pathophysiological mechanism between BPV and cSVD might involve a higher degree of mechanical stress on vessel walls leading to endothelial injury, arterial stiffness, and ischemic hypoperfusion. A bidirectional association is also plausible, i.e., cSVD may be associated with neurodegenerative changes to cortical structures that are involved in central control of $\mathrm{BP}$ and heart rate, leading to high BPV [45].

Determinants of BPV are still under investigation, but it is likely that these differ between different measurement methods and timeframes of BPV [22]. This idea is strengthened by the observation that there is only a very weak correlation between visit-to-visit, 24-h and day-to-day BPV [46]. Short-term variability seems mainly determined by physiological mechanisms, including the baroreflex, humoral factors, 
and the circadian rhythm. For long-term variability, behavioral factors, compliance to antihypertensive treatment and seasonal changes are involved as well. Despite these differences, all types of BPV have now been linked to cSVD. Given the challenges in determining visit-to-visit BPV, which requires multiple clinic visits, and the preference of older people with cognitive impairment for HBPM over 24-h BP measurements [47], day-to-day BPV may be a more feasible approach to assess BPV in relation to cognition and CSVD.

In our study, BPV was lower in the morning compared to the evening. This has been reported before [48]. When we differentiated between morning and evening BP values, only BP and BPV in the evening were associated with cSVD. Lower within-subject variation in morning measurements can be expected, given that these are less affected by physical activity, stress, and other factors that are more likely to occur during the course of the day. Also, circumstances of the measurement taken in the morning are potentially more standardized (after waking up, but before medication intake and breakfast) than in the evening (before or at least $1 \mathrm{~h}$ after dinner, after taking 5 min of rest) [49]. It might therefore be the case that the range of morning BPV was too small to detect significant associations with cSVD.

This study has several strengths and limitations. Compared to previous studies on BPV and CSVD, we had a relatively small sample size. However, we were able to study a combination of four markers of cSVD instead of focusing on one or two and we studied BPV from day-to-day. Our study population was based on older people referred to a memory clinic. This comprises a group in which (subjective) cognitive impairment is present and who have different diagnoses and mixed pathologies. The use of convenience sample might have introduced selection bias and limits generalizability. Moreover, only patients in whom an MRI was deemed informative for clinical decision making were included and patients had to be willing and able to perform HBPM. We used international criteria for the assessment of cSVD markers, with moderate to excellent interrater agreement [1]. Although the total cSVD score as proposed by Staals and colleagues is a valid measure to estimate total brain damage on MRI, the content validity of the scale might be improved by taking into account location and number of individual markers [28]. A limitation of the current study is the use of two MR scanners. Although this may have been a source of bias, the mean cSVD scores were similar (Supplementary
Table 2). In addition, this is more likely to represent clinical reality.

In conclusion, we found that BPV from day-to-day was associated with cSVD burden, independent from mean BP, in older people attending a memory clinic. This study provides the field with new information regarding BPV and cSVD. Future longitudinal studies in a population-based sample are warranted to assess the effect of day-to-day BPV on progression of cSVD and the potential of modifying day-to-day $\mathrm{BPV}$ in order to reduce $\mathrm{cSVD}$ progression.

\section{ACKNOWLEDGMENTS}

We are grateful to all participants and their caregivers for their effort. We would like to thank the secretary and all involved residents of the Radboudumc Alzheimer Center for their support with the recruitment and are grateful to Anne Lieke Meendering, Lotte van den Ingh, Faize Önen, Emma Peters, Sara Barmentloo and Cynthia ter Horst for their role in data capturing. This study was funded by the Dutch Alzheimer Society (grant number WE.09-2015-03).

Authors' disclosures available online (https:// www.j-alz.com/manuscript-disclosures/19-1134r2).

\section{SUPPLEMENTARY MATERIAL}

The supplementary Table is available in the electronic version of this article: https://dx.doi.org/ 10.3233/JAD-191134.

\section{REFERENCES}

[1] Wardlaw JM, Smith EE, Biessels GJ, Cordonnier C, Fazekas F, Frayne R, Lindley RI, O'Brien JT, Barkhof F, Benavente OR, Black SE, Brayne C, Breteler M, Chabriat H, Decarli C, de Leeuw FE, Doubal F, Duering M, Fox NC, Greenberg S, Hachinski V, Kilimann I, Mok V, Oostenbrugge R, Pantoni L, Speck O, Stephan BC, Teipel S, Viswanathan A, Werring D, Chen C, Smith C, van Buchem M, Norrving B, Gorelick PB, Dichgans M (2013) Neuroimaging standards for research into small vessel disease and its contribution to ageing and neurodegeneration. Lancet Neurol 12, 822-838.

[2] Vernooij MW, van der Lugt A, Ikram MA, Wielopolski PA, Niessen WJ, Hofman A, Krestin GP, Breteler MMB (2008) Prevalence and risk factors of cerebral microbleeds: The Rotterdam Scan Study. Neurology 70, 1208-1214.

[3] de Leeuw FE, de Groot JC, Achten E, Oudkerk M, Ramos LM, Heijboer R, Hofman A, Jolles J, van Gijn J, Breteler MM (2001) Prevalence of cerebral white matter lesions in elderly people: A population based magnetic resonance imaging study. The Rotterdam Scan Study. J Neurol Neurosurg Psychiatry 70, 9-14.

[4] Pantoni L, Garcia JH (1997) Pathogenesis of leukoaraiosis: A review. Stroke 28, 652-659. 
[5] Gorelick PB, Scuteri A, Black SE, DeCarli C, Greenberg SM, Iadecola C, Launer LJ, Laurent S, Lopez OL, Nyenhuis D, Petersen RC, Schneider JA, Tzourio C, Arnett DK, Bennett DA, Chui HC, Higashida RT, Lindquist R, Nilsson PM, Roman GC, Sellke FW, Seshadri S (2011) Vascular contributions to cognitive impairment and dementia. Stroke 42, 2672-2713.

[6] Schneider JA, Arvanitakis Z, Bang W, Bennett DA (2007) Mixed brain pathologies account for most dementia cases in community-dwelling older persons. Neurology 69, 21972204.

[7] Bezerra DC, Sharrett AR, Matsushita K, Gottesman RF, Shibata D, Mosley TH Jr., Coresh J, Szklo M, Carvalho MS, Selvin E (2012) Risk factors for lacune subtypes in the Atherosclerosis Risk in Communities (ARIC) Study. Neurology 78, 102-108.

[8] van Dijk EJ, Breteler MM, Schmidt R, Berger K, Nilsson LG, Oudkerk M, Pajak A, Sans S, de Ridder M, Dufouil C, Fuhrer R, Giampaoli S, Launer LJ, Hofman A (2004) The association between blood pressure, hypertension, and cerebral white matter lesions: Cardiovascular determinants of dementia study. Hypertension 44, 625-630.

[9] Cordonnier C, Al-Shahi Salman R, Wardlaw J (2007) Spontaneous brain microbleeds: Systematic review, subgroup analyses and standards for study design and reporting. Brain 130, 1988-2003.

[10] Yakushiji Y, Charidimou A, Hara M, Noguchi T, Nishihara M, Eriguchi M, Nanri Y, Nishiyama M, Werring DJ, Hara H (2014) Topography and associations of perivascular spaces in healthy adults: The Kashima scan study. Neurology 83, 2116-2123.

[11] Wardlaw JM, Smith C, Dichgans M (2013) Mechanisms of sporadic cerebral small vessel disease: Insights from neuroimaging. Lancet Neurol 12, 483-497.

[12] Iadecola C, Yaffe K, Biller J, Bratzke LC, Faraci FM, Gorelick PB, Gulati M, Kamel H, Knopman DS, Launer LJ, Saczynski JS, Seshadri S, Zeki Al Hazzouri A (2016) Impact of hypertension on cognitive function: A scientific statement from the American Heart Association. Hypertension 68, e67-e94.

[13] Kivipelto M, Helkala EL, Laakso MP, Hanninen T, Hallikainen M, Alhainen K, Soininen H, Tuomilehto J, Nissinen A (2001) Midlife vascular risk factors and Alzheimer's disease in later life: Longitudinal, population based study. BMJ 322, 1447-1451.

[14] Mossello E, Pieraccioli M, Nesti N, Bulgaresi M, Lorenzi C, Caleri V, Tonon E, Cavallini MC, Baroncini C, Di Bari M, Baldasseroni S, Cantini C, Biagini CA, Marchionni N, Ungar A (2015) Effects of low blood pressure in cognitively impaired elderly patients treated with antihypertensive drugs. JAMA Intern Med 175, 578-585.

[15] Walker KA, Power MC, Gottesman RF (2017) Defining the relationship between hypertension, cognitive decline, and dementia: A review. Curr Hypertens Rep 19, 24.

[16] Rothwell PM (2010) Limitations of the usual blood-pressure hypothesis and importance of variability, instability, and episodic hypertension. Lancet 375, 938-948.

[17] Rothwell PM, Howard SC, Dolan E, O'Brien E, Dobson JE, Dahlof B, Sever PS, Poulter NR (2010) Prognostic significance of visit-to-visit variability, maximum systolic blood pressure, and episodic hypertension. Lancet 375, 895-905.

[18] Muntner P, Shimbo D, Tonelli M, Reynolds K, Arnett DK, Oparil S (2011) The relationship between visit-to-visit variability in systolic blood pressure and all-cause mortality in the general population. Hypertension 57, 160-166.
[19] Tai C, Sun Y, Dai N, Xu D, Chen W, Wang J, Protogerou A, van Sloten TT, Blacher J, Safar ME, Zhang Y, Xu Y (2015) Prognostic significance of visit-to-visit systolic blood pressure variability: A meta-analysis of 77,299 patients. J Clin Hypertens (Greenwich) 17, 107-115.

[20] Brickman AM, Reitz C, Luchsinger JA, Manly JJ, Schupf N, Muraskin J, DeCarli C, Brown TR, Mayeux R (2010) Long-term blood pressure fluctuation and cerebrovascular disease in an elderly cohort. Arch Neurol 67, 564-569.

[21] Tully PJ, Debette S, Tzourio C (2018) The association between systolic blood pressure variability with depression, cognitive decline and white matter hyperintensities: The 3C Dijon MRI study. Psychol Med 48, 1444-1453.

[22] Parati G, Ochoa JE, Lombardi C, Bilo G (2013) Assessment and management of blood-pressure variability. Nat Rev Cardiol 10, 143-155.

[23] Stergiou GS, Parati G, Vlachopoulos C, Achimastos A, Andreadis E, Asmar R, Avolio A, Benetos A, Bilo G, Boubouchairopoulou N, Boutouyrie P, Castiglioni P, de la Sierra A, Dolan E, Head G, Imai Y, Kario K, Kollias A, Kotsis V, Manios E, McManus R, Mengden T, Mihailidou A, Myers M, Niiranen T, Ochoa JE, Ohkubo T, Omboni S, Padfield P, Palatini P, Papaioannou T, Protogerou A, Redon J, Verdecchia P, Wang J, Zanchetti A, Mancia G, O'Brien E (2016) Methodology and technology for peripheral and central blood pressure and blood pressure variability measurement: Current status and future directions - Position statement of the European Society of Hypertension Working Group on blood pressure monitoring and cardiovascular variability. J Hypertens 34, 1665-1677.

[24] Liu Z, Zhao Y, Zhang H, Chai Q, Cui Y, Diao Y, Xiu J, Sun X, Jiang G (2015) Excessive variability in systolic blood pressure that is self-measured at home exacerbates the progression of brain white matter lesions and cognitive impairment in the oldest old. Hypertens Res 39, 245-253.

[25] Yang S, Qin W, Yang L, Fan H, Li Y, Yin J, Hu W (2017) The relationship between ambulatory blood pressure variability and enlarged perivascular spaces: A cross-sectional study. BMJ Open 7, e015719.

[26] Yang S, Yuan J, Qin W, Yang L, Fan H, Li Y, Hu W (2018) Twenty-four-hour ambulatory blood pressure variability is associated with total magnetic resonance imaging burden of cerebral small-vessel disease. Clin Interv Aging 13, 14191427.

[27] Huijts M, Duits A, van Oostenbrugge RJ, Kroon AA, de Leeuw PW, Staals J (2013) Accumulation of MRI markers of cerebral small vessel disease is associated with decreased cognitive function. A study in first-ever lacunar stroke and hypertensive patients. Front Aging Neurosci 5, 72.

[28] Staals J, Makin SD, Doubal FN, Dennis MS, Wardlaw JM (2014) Stroke subtype, vascular risk factors, and total MRI brain small-vessel disease burden. Neurology 83, 12281234.

[29] Banerjee G, Jang H, Kim HJ, Kim ST, Kim JS, Lee JH, Im K, Kwon H, Lee JM, Na DL, Seo SW, Werring DJ (2018) Total MRI small vessel disease burden correlates with cognitive performance, cortical atrophy, and network measures in a memory clinic population. J Alzheimers Dis 63, 1485-1497.

[30] Meijs AP, Claassen JA, Rikkert MG, Schalk BW, Meulenbroek O, Kessels RP, Melis RJ (2015) How does additional diagnostic testing influence the initial diagnosis in patients with cognitive complaints in a memory clinic setting? Age Ageing 44, 72-77.

[31] Stergiou GS, Giovas PP, Gkinos CP, Patouras JD (2007) Validation of the Microlife WatchBP Home device for self home 
blood pressure measurement according to the International Protocol. Blood Press Monit 12, 185-188.

[32] Parati G, Stergiou GS, Asmar R, Bilo G, de Leeuw P, Imai Y, Kario K, Lurbe E, Manolis A, Mengden T, O’Brien E, Ohkubo T, Padfield P, Palatini P, Pickering T, Redon J, Revera M, Ruilope LM, Shennan A, Staessen JA, Tisler A, Waeber B, Zanchetti A, Mancia G (2008) European Society of Hypertension guidelines for blood pressure monitoring at home: A summary report of the Second International Consensus Conference on Home Blood Pressure Monitoring. $J$ Hypertens 26, 1505-1526.

[33] Fazekas F, Chawluk JB, Alavi A, Hurtig HI, Zimmerman RA (1987) MR signal abnormalities at 1.5 T in Alzheimer's dementia and normal aging. Am J Roentgenol 149, 351-356.

[34] Gregoire SM, Chaudhary UJ, Brown MM, Yousry TA, Kallis C, Jager HR, Werring DJ (2009) The Microbleed Anatomical Rating Scale (MARS): Reliability of a tool to map brain microbleeds. Neurology 73, 1759-1766.

[35] Doubal FN, MacLullich AM, Ferguson KJ, Dennis MS, Wardlaw JM (2010) Enlarged perivascular spaces on MRI are a feature of cerebral small vessel disease. Stroke 41, 450-454.

[36] Petersen RC (2004) Mild cognitive impairment as a diagnostic entity. J Intern Med 256, 183-194.

[37] American Psychiatric Association (2000) Diagnostic and statistical manual of mental disorders (4th ed., text rev.). American Psychiatric Association Publishing, Washington, DC.

[38] Yilmaz P, Ikram MK, Niessen WJ, Ikram MA, Vernooij MW (2018) Practical small vessel disease score relates to stroke, dementia, and death. Stroke 49, 2857-2865.

[39] Haring B, Liu J, Salmoirago-Blotcher E, Hayden KM, Sarto G, Roussouw J, Kuller LH, Rapp SR, Wassertheil-Smoller S (2019) Blood pressure variability and brain morphology in elderly women without cardiovascular disease. Neurology 92, e1284-e1297.

[40] Oishi E, Ohara T, Sakata S, Fukuhara M, Hata J, Yoshida D, Shibata M, Ohtsubo T, Kitazono T, Kiyohara Y, Ninomiya $\mathrm{T}$ (2017) Day-to-day blood pressure variability and risk of dementia in a general Japanese elderly population: The Hisayama Study. Circulation 136, 516-525.

[41] Matsumoto A, Satoh M, Kikuya M, Ohkubo T, Hirano M, Inoue R, Hashimoto T, Hara A, Hirose T, Obara T, Metoki H,
Asayama K, Hosokawa A, Totsune K, Hoshi H, Hosokawa T, Sato H, Imai Y (2014) Day-to-day variability in home blood pressure is associated with cognitive decline: The Ohasama study. Hypertension 63, 1333-1338.

[42] de Heus RAA, Olde Rikkert MGM, Tully PJ, Lawlor BA, Claassen J (2019) Blood pressure variability and progression of clinical Alzheimer disease. Hypertension 74, 1172-1180.

[43] O'Caoimh R, Gao Y, Svendrovski A, Illario M, Iaccarino G, Yavuz BB, Kehoe PG, Molloy DW (2019) Effect of visit-to-visit blood pressure variability on cognitive and functional decline in mild to moderate Alzheimer's disease. J Alzheimers Dis 68, 1499-1510.

[44] Zhou TL, Kroon AA, van Sloten TT, van Boxtel MPJ, Verhey FRJ, Schram MT, Kohler S, Stehouwer CDA, Henry RMA (2019) Greater blood pressure variability is associated with lower cognitive performance. Hypertension 73, 803-811.

[45] Palatini P (2014) Day-by-day blood pressure variability: Cause or consequence of vascular brain injury? Hypertension 63, 1163-1165.

[46] Juhanoja EP, Niiranen TJ, Johansson JK, Puukka PJ, Jula AM (2016) Agreement between ambulatory, home, and office blood pressure variability. J Hypertens 34, 61-67.

[47] Plichart M, Seux ML, Caillard L, Chaussade E, Vidal JS, Boully C, Hanon O (2013) Home blood pressure measurement in elderly patients with cognitive impairment: Comparison of agreement between relative-measured blood pressure and automated blood pressure measurement. Blood Press Monit 18, 208-214.

[48] Imai Y, Nishiyama A, Sekino M, Aihara A, Kikuya M, Ohkubo T, Matsubara M, Hozawa A, Tsuji I, Ito S, Satoh H, Nagai K, Hisamichi S (1999) Characteristics of blood pressure measured at home in the morning and in the evening: The Ohasama study. J Hypertens 17, 889-898.

[49] Williams B, Mancia G, Spiering W, Agabiti Rosei E, Azizi M, Burnier M, Clement DL, Coca A, de Simone G, Dominiczak A, Kahan T, Mahfoud F, Redon J, Ruilope L, Zanchetti A, Kerins M, Kjeldsen SE, Kreutz R, Laurent S, Lip GYH, McManus R, Narkiewicz K, Ruschitzka F, Schmieder RE, Shlyakhto E, Tsioufis C, Aboyans V, Desormais I, ESC Scientific Document Group (2018) ESC/ESH Guidelines for the management of arterial hypertension. Eur Heart J 39, 3021-3104. 